\title{
Role of IncRNA FTX in invasion, metastasis, and epithelial- mesenchymal transition of endometrial stromal cells caused by endometriosis by regulating the PI3K/Akt signaling pathway
}

\author{
Huixiang Wang, Chengxiang Ni, Wei Xiao, Sulin Wang \\ Department of Gynecology and Obstetrics, Beijing Tongren Hospital, Capital Medical University, Beijing, China \\ Contributions: (I) Conception and design: H Wang; (II) Administrative support: H Wang; (III) Provision of study materials or patients: W Xiao, S \\ Wang; (IV) Collection and assembly of data: H Wang, C Ni; (V) Data analysis and interpretation: H Wang; (VI) Manuscript writing: All authors; (VII) \\ Final approval of manuscript: All authors. \\ Correspondence to: Huixiang Wang. Department of Gynecology and Obstetrics, Beijing Tongren Hospital, Capital Medical University, Beijing, China. \\ Email: danhong151@163.com.
}

Background: Studies have considered long non-coding RNA 5 prime to Xist (lncRNA FTX) a key lncRNA for normal uterine development, but it has not been reported whether lncRNA FTX is involved in regulating the development of endometriosis (EMs). The aim of the present study was to explore the effect and mechanism of long non-coding RNA 5 prime to Xist (lncRNA FTX) on the invasion, metastasis, and epithelial-mesenchymal transition (EMT) of endometrial stromal cells (ESCs) caused by EMs.

Methods: Ectopic or normal endometrial tissues were collected from 38 patients with EMs, who were diagnosed and operated on at Beijing Tongren Hospital from June 2018 to December 2019, and 20 healthy volunteers with normal endometria. The expression of lncRNA FTX in both groups was detected by quantitative reverse transcription polymerase chain reaction. Ectopic endometrial stromal cells (EESC) and ESC from patients with EMs and healthy volunteers were separated and cultured, and the expression of lncRNA FTX in the cells was detected. The expression of lncRNA FTX in EESC was overexpressed or interfered. Proliferation, invasion, and migration was detected by Cell Counting Kit-8, transwell assay, and scratch assay. Apoptosis and cell cycle were detected by flow cytometry. EMT-related protein and PI3K/Akt signaling pathway-related protein expressions were detected by Western blot.

Results: LncRNA FTX was underexpressed in endometrial tissues and EESC from patients with EMs. The overexpression of IncRNA FTX could significantly inhibit the proliferation, invasion, and migration of EESC, but promoted apoptosis and cell cycle arrest in the G0/G1 phase. The overexpression of lncRNA FTX significantly increased the expression of EMT-related protein, E-cadherin, and decreased the protein expression of vimentin, N-cadherin, and zinc finger E-box binding homeobox 1 . In addition, the overexpression of lncRNA FTX could decrease the expression of $\mathrm{p}-\mathrm{PI} 3 \mathrm{~K} / \mathrm{PI} 3 \mathrm{~K}$ and p-Akt/Akt. Interfering with the expression of lncRNA FTX had the opposite result.

Conclusions: The overexpression of lncRNA FTX could decrease the invasion, metastasis, and EMT of ESC caused by EMs by inhibiting the activity of the PI3K/Akt signaling pathway.

Keywords: Endometriosis (EMs); endometrial stromal cells (ESCs); long non-coding RNA 5 prime to Xist; epithelial-mesenchymal transition (EMT); PI3K/Akt signaling pathway

Submitted Sep 04, 2020. Accepted for publication Nov 11, 2020.

doi: $10.21037 / \mathrm{atm}-20-6810$

View this article at: http://dx.doi.org/10.21037/atm-20-6810 


\section{Introduction}

The main symptoms of endometriosis (EMs) are pelvic pain and infertility caused by the implantation and growth of endometrial tissues with growth function outside the uterine cavity (1), which have serious adverse effects on the health and fertility of women of reproductive age (2). It has been reported that the incidence of EMs has significantly increased in recent years, affecting 10-15\% of women of reproductive age; the incidence is as high as $25-40 \%$ among infertile women (3). It has been noted that ectopic endometrial tissues and cells have biologic functions of abnormal proliferation, invasion, metastasis, and apoptosis resistance, which are similar to malignant tumor cells $(4,5)$. EMs is often treated by surgery in clinical practice, but is a difficult disease to cure, as it involves the thorough removal of tiny ectopic endometria. Because the pathogenesis of EMs remains unclear and its etiology cannot be completely eliminated, it is prone to recurrent attacks after surgery. Therefore, a better understanding of the pathogenesis of EMs is a priority.

Many studies have found that long non-coding RNAs (lncRNAs) cause a variety of biologic behaviors in EMs, which may be a key factor in the diagnosis or treatment of EMs $(6,7)$. IncRNAs are a type of RNA molecule; they are longer than $200 \mathrm{nt}$ and do not encode proteins (8) lncRNAs not only participate in the physiological process of body growth and development, but also play a crucial role in the incidence and development of diseases (9). Most IncRNAs cannot only regulate DNA replication, RNA transcription, and protein translation by complementary pairing with microRNAs or circRNAs, but also directly interact with target proteins to regulate protein activity (10). It has been noted that inhibiting the expression of lncRNA BRAF-activated non-coding RNA may inhibit the development of ectopic endometrial tissues by inhibiting the extracellular signal-regulated kinase/mitogen-activated protein kinase signaling pathway (11). Additionally, increasing the expression of lncRNA urothelial carcinoma associated 1 has the potential to reduce the risk of EMs (12). Therefore, lncRNA is important for regulating EMs development. Some studies have considered long noncoding RNA 5 prime to Xist (lncRNA FTX) a key lncRNA for normal uterine development (13), but it has not been reported whether lncRNA FTX is involved in regulating the development of EMs, and most of the previous studies on the relationship between lncrnas and endometriosis were conducted independently of clinical samples, or only explored the relationship between lncrnas expression and disease development. Therefore, based on the expression level of clinical samples, in the present study, we aimed to explore the effect and its mechanism of lncRNA FTX on the invasion, metastasis, and epithelial-mesenchymal transition (EMT) of endometrial stromal cells (ESC) caused by EMs in vitro so as to provide a theoretical basis for the diagnosis and treatment of EMs to find new therapeutic targets. We present the following article in accordance with the MDAR reporting checklist (available at http://dx.doi. org/10.21037/atm-20-6810).

\section{Methods}

\section{Collection of tissue samples and clinical data}

Patients with EMs who were diagnosed and surgically treated at Beijing Tongren Hospital from June 2018 to December $2019(\mathrm{n}=38)$ and healthy volunteers without EMs who underwent physical examination at our hospital during this period $(n=20)$ were included the present study. The age of the included patients was recorded. Patients with the following conditions were included as samples: patients diagnosed with EMs by B-ultrasound and histopathology; patients who had not received hormone therapy within 3 months; patients with normal function of vital organs, such as heart, lung, liver, and kidney; and patients with normal menstruation. Patients with the following conditions were excluded: patients with complicated endocrine, autoimmune, and coagulation disorders and mental disorders; patients with gynecological diseases, such as complicated hysteromyoma and polycystic ovary syndrome; and lactating women, pregnant women, and women who were possibly pregnancy. All study procedures were approved by the Research Ethics Committee of Beijing Tongren Hospital. All procedures performed in this study involving human participants were in accordance with the Declaration of Helsinki (as revised in 2013). Informed consent was taken from all the patients.

\section{Cell culture and transfection}

After removal of normal or ectopic endometrial tissue under aseptic conditions, the tissues were cut into $1-\mathrm{mm}^{3}$ pieces, transferred to phosphate-buffered saline (PBS) containing $300 \mathrm{mg} / \mathrm{mL}$ of collagenase III and $40 \mathrm{mg} / \mathrm{mL}$ of deoxyribonuclease, and digested at $37{ }^{\circ} \mathrm{C}$ with oscillation at $2.5 \mathrm{~g}$. After $45 \mathrm{~min}$, the digestive juice was filtered through a 
Table 1 Primers for quantification

\begin{tabular}{ll}
\hline RNA & Sequences $\left(5^{\prime}-3^{\prime}\right)$ \\
\hline IncRNA FTX & Forward: 5'-GTGTCTCTCTCTCTCTCTCTCTT-3' \\
& Reverse: 5'-CCTCTTCAGCAGTAGCATAGTT-3' \\
GAPDH & Forward: 5'-TCCCATCACCATCTTCCA-3' \\
& Reverse: 5'-CATCACGCCACAGTTTCC-3' \\
\hline
\end{tabular}

LncRNA FTX, long non-coding RNA 5 prime to Xist; GAPDH, glyceraldehyde-3-phosphate dehydrogenase.

$40-\mu \mathrm{m}$ filter to remove the undigested tissues. The digested individual cells were separated and collected into centrifuge tubes, and the digestion was terminated by adding fetal bovine serum (FBS; Gibco, USA). After centrifuging at $800 \mathrm{rpm}$ for $5 \mathrm{~min}$ at $4{ }^{\circ} \mathrm{C}$, the supernatant was discarded, and F12 medium containing $10 \%$ serum was added to the resuspended cells. The cells were seeded in a $25-\mathrm{cm}^{2}$ culture flask and placed in a $37{ }^{\circ} \mathrm{C}, 5 \% \mathrm{CO}_{2}$ incubator (Thermo Fisher Scientific, Waltham, USA) to culture. ESC and ectopic endometrial stromal cells (EESC) were obtained. EESC were transfected with negative siRNA (si-NC), lncRNA FTX siRNA (si-FTX), pcDNA3.1 empty vector (pc-NC), and overexpressed pcDNA3.1-lncRNA FTX (pc-FTX) following the Lipofectamine 2000 transfection procedure of (Thermo, USA). EESC without any treatment were used as the control group. LncRNA FTX siRNA was designed and synthesized by Ruibo Bio (Guangzhou, China). The overexpressed pcDNA3.1-lncRNA FTX plasmid was synthesized by Addgene (USA).

\section{Quantitative reverse transcription polymerase chain reaction (qRT-PCR) assay}

Total RNA was extracted from tissues and cells using the TRIzol method (Thermo Fisher Scientific, Waltham, USA). The concentration and purity of RNA were detected with NanoDrop (Thermo Fisher Scientific, Waltham, USA). Reverse transcription into cDNA was performed following the random primer reverse transcription kit (Thermo, USA). The expression level of lncRNA FTX was detected following the instructions of the SYBR Green kit (TaKaRa, Japan). Six replicates were set up for the experiment using glyceraldehyde-3-phosphate dehydrogenase (GAPDH) as an internal control. The experimental data obtained by qRT-PCR were used to calculate the relative expression of the target gene using the $2^{-\Delta \Delta \mathrm{Ct}}$ method. Primer sequences are shown in Table 1.

\section{Cell proliferation rate detection by Cell Counting Kit-8 (CCK8)}

A total of $5 \times 10^{4}$ EESC were seeded onto opaque 96-well plates, supplemented with $200 \mu \mathrm{L}$ fresh F12 complete medium for $24 \mathrm{~h}$. After the transfection of cells was completed, the cells were incubated for a further $24 \mathrm{~h}$. After incubation, the fresh medium was changed, and the cells were cultured for 24,48 , and $72 \mathrm{~h}$, respectively. A total of $10 \mu \mathrm{L}$ CCK8 was added to each well, and the cells were incubated in the incubator for $1.5 \mathrm{~h}$. The absorbance value (optical density) was measured with a microplate reader $(450 \mathrm{~nm})$.

\section{Cell invasion detection by transwell assay}

Twenty-four hours after transfection with $2 \times 10^{4}$ cells added to the upper chamber of the transwell chamber, $700 \mu \mathrm{L}$ medium containing $20 \%$ FBS was added to the lower chamber of the transwell chamber. The transwell chamber was removed after $12-24 \mathrm{~h}$ of culture at $37^{\circ} \mathrm{C}, 5 \% \mathrm{CO}_{2}$, and was washed 3 times with PBS. The chamber was fixed with $1 \%$ glutaraldehyde for $30 \mathrm{~min}$, washed with PBS, and dried; $0.1 \%$ crystal violet was then added and left for $12 \mathrm{~h}$. The transwell chamber was then washed with PBS again. After the transwell chamber was dried, an upright microscope was used to observe 6-10 random fields. The number of positive cells in each field was recorded, and 3 fields were randomly chosen for photography and statistical analysis.

\section{Cell migration detection by scratch assay}

First, a transverse line was drawn with a ruler behind the 6-well plates with a marker, and then the cells transfected for $24 \mathrm{~h}$ were seeded onto the 6 -well plates. After the cells were transfected separately, a $10 \mu \mathrm{L}$ sterile lance tip was placed against a ruler and used to scratch perpendicular to the transverse line. Suspended cells and cell debris were removed by washing with PBS. Fresh serum-free medium was added, and after $24 \mathrm{~h}$ of culture, photographs were taken with an inverted microscope to record the experimental results. Image J software (National Institutes of Health, Bethesda, Maryland, USA) was used to calculate the scratch area.

\section{Apoptosis detection by flow cytometry}

Cells that had been transfected for $24 \mathrm{~h}$ were digested into centrifuge tubes using trypsin. After centrifugation at $71 \mathrm{~g}$ for $5 \mathrm{~min}$ at $4{ }^{\circ} \mathrm{C}$, the cells were rinsed twice with pre- 
chilled sterile PBS. After centrifugation at $71 \mathrm{~g}$ for $5 \mathrm{~min}$ at $4{ }^{\circ} \mathrm{C}$, the cell concentration was adjusted to $5 \times 10^{5}$ cells $/ \mathrm{mL}$ with PBS. A total of $200 \mu \mathrm{L}$ cell suspension was taken and added with $10 \mu \mathrm{L}$ Annexin $\mathrm{V}$-fluorescein isothiocyanate and $10 \mu \mathrm{L}$ propidium iodide (PI) solution at a concentration of $20 \mathrm{mg} / \mathrm{L}$. This mixture was incubated for $10 \mathrm{~min}$ at room temperature under dark conditions. After adding $500 \mu \mathrm{L}$ PBS, apoptosis was detected by flow cytometry.

\section{Cell cycle detection by flow cytometry}

Cells that had been transfected for $24 \mathrm{~h}$ were digested into centrifuge tubes using trypsin. After centrifugation at $71 \mathrm{~g}$ for $5 \mathrm{~min}$ at $4{ }^{\circ} \mathrm{C}$, the cells were rinsed twice with prechilled sterile PBS. After centrifugation at $71 \mathrm{~g}$ for $5 \mathrm{~min}$ at $4{ }^{\circ} \mathrm{C}$, the cells were resuspended by adding $1 \mathrm{~mL}$ pre-chilled ethanol with a volume fraction of $70 \%$, and fixed overnight at $4{ }^{\circ} \mathrm{C}$. After centrifugation, the cells were rinsed twice with pre-chilled sterile PBS. The cells were centrifuged for 5 min each time, and resuspended in $500 \mu \mathrm{L}$ PBS. After adding PI solution with a final concentration of $50 \mu \mathrm{g} / \mathrm{mL}$, the cells were incubated at room temperature for $15 \mathrm{~min}$ under dark conditions. The cell cycle was detected by flow cytometry.

\section{Protein expression detection by Western blot}

Cells that had been transfected for $24 \mathrm{~h}$ were rinsed twice with pre-chilled sterile PBS to remove PBS, and $80 \mu \mathrm{L}$ cell lysate was added for $15 \mathrm{~min}$ at $4{ }^{\circ} \mathrm{C}$. The lysed cells were transferred to a $200 \mu \mathrm{L}$ centrifuge tube at a centrifugation of $15,984 \times \mathrm{g}$ for $15 \mathrm{~min}$ at $4{ }^{\circ} \mathrm{C}$; the cellular protein was then collected. The protein concentration was determined with a bicinchoninic acid (BCA) kit. In total, $20 \mu \mathrm{g}$ protein was denatured by boiling in $1 \times$ loading buffer, and proteins were separated by sodium dodecyl sulfatepolyacrylamide gel electrophoresis. Proteins were then transferred to polyvinylidene difluoride membranes; $5 \%$ skimmed milk powder was then added to block for $1 \mathrm{~h}$. Primary antibodies E-cadherin (Abcam, UK), vimentin (Abcam, UK), N-cadherin (Abcam, UK), zinc finger E-box binding homeobox 1 (ZEB1; Abcam, UK), p-PI3K (Abcam, UK), PI3K (Abcam, UK), p-Akt (Abcam, UK) and Akt (Abcam, UK) were added and later incubated overnight at $4{ }^{\circ} \mathrm{C}$. The membranes were washed 3 times, and secondary antibodies were added to incubate for $1 \mathrm{~h}$ in the greenhouse. After washing the membrane a further 3 times, chemiluminescence reagents were added to develop the protein. Images were collected in a gel imaging system. Gray levels of the protein bands were analyzed using Image J software, with GAPDH as an internal reference to calculate the relative protein expression.

\section{Statistical analysis}

SPSS version 24.0 (SPSS, Chicago, IL, USA) was used for one-way analysis of variance and independent sample $t$-test analysis. The results were expressed as mean \pm standard deviation. $\mathrm{P}<0.05$ indicated statistical significance.

\section{Results}

\section{lncRNA FTX underexpression in endometrial tissues and cells}

To determine the expression of lncRNA FTX in EMs, we collected endometrial tissues from patients with EMs and healthy volunteers. The expression of lncRNA FTX was found to be significantly reduced in the endometrial tissues of patients with EMs by qRT-PCR detection of clinical samples compared with the normal group (Figure $1 A$ ). Furthermore, we separated and cultured ESC and EESC. Compared with the ESC group, the expression level of lncRNA FTX was significantly reduced in the EESC group (Figure 1B). To further investigate the functional impact of the expression of lncRNA FTX on EESC, we overexpressed and interfered with the expression of lncRNA FTX in EESC. The results of the qRT-PCR detection indicated that the expression level of lncRNA FTX was significantly decreased in the cells of the si-FTX group compared with the si-NC group (Figure 1C). The expression level of lncRNA FTX was significantly increased in the cells of the pc-FTX group compared with the pc-NC group.

\section{lncRNA FTX promotes proliferation, invasion, and migration of EESC}

CCK8, transwell assay, and scratch assay were used to investigate the effect of lncRNA FTX on proliferation, invasion, and migration of EESC, respectively. According to the results of CCK8 assay (Figure $2 A$ ), the proliferation level of EESC was significantly increased after interfering with the expression of lncRNA FTX $(\mathrm{P}<0.05)$, and the proliferation level of EESC was significantly reduced after the overexpression of $\operatorname{lncRNA}$ FTX $(\mathrm{P}<0.05)$. The results of the transwell assay showed that the invasion of cells in the si-FTX group was significantly increased compared with 

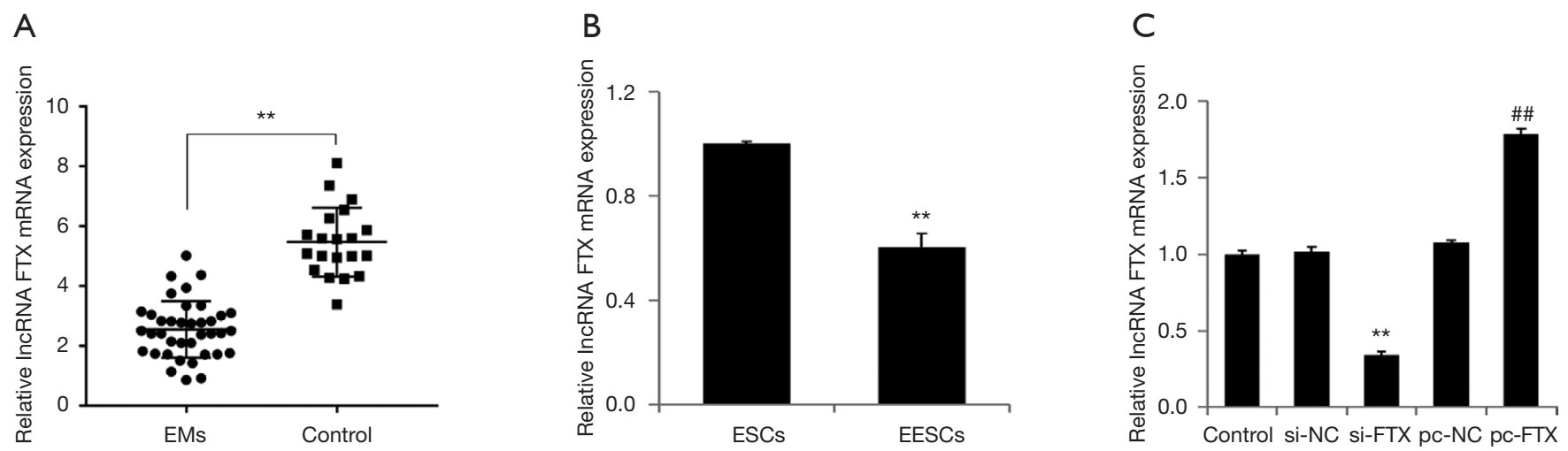

Figure 1 Long non-coding RNA 5 prime to Xist (lncRNA FTX) was underexpressed in endometriotic tissues and cells. (A) Quantitative reverse transcription polymerase chain reaction (qRT-PCR) was used to detect the expression of lncRNA FTX in the endometrial tissues of patients with endometriosis and in healthy volunteers; (B) qRT-PCR was used to detect the expression of lncRNA FTX in endometrial stroma cells (ESCs) and ectopic endometrial stromal cells (EESCs); (C) qRT-PCR was used to verify the expression of lncRNA FTX in EESC after transfection. **, compared with negative siRNA (si-NC) group, and $\mathrm{P}<0.01$; ${ }^{\# \#}$, compared with pcDNA3.1 empty vector (pc-NC) group, and $\mathrm{P}<0.01$. EMs, endometriosis; si-NC, EESC transfected with negative siRNA; si-FTX, lncRNA FTX siRNA; pc-NC, pcDNA3.1 empty vector; pc-FTX, overexpressed pcDNA3.1-lncRNA FTX.

the si-NC group $(\mathrm{P}<0.05)$, and the invasion of cells in the pc-FTX group was significantly decreased compared with the pc-NC group $(\mathrm{P}<0.05)$ (Figure $2 B)$. The results of the scratch assay showed that the migration of cells in the siFTX group was significantly increased compared with the si-NC group $(\mathrm{P}<0.05)$, and the migration of cells in the pcFTX group was significantly decreased compared with the pc-NC group $(\mathrm{P}<0.05)$ (Figure $2 C)$.

\section{LncRNA FTX inbibits apoptosis in EESC and causes cell cycle arrest in the $S$ phase}

The flow cytometry results showed that the apoptotic rate of EESC significantly decreased after interfering with the expression of lncRNA FTX (Figure 3A). The proportion of cells in the G0/G1 phase significantly decreased, whereas the proportion of cells in the $\mathrm{S}$ phase increased (Figure 3B). The apoptotic rate of cells significantly increased after the overexpression of 1ncRNA FTX (Figure 3A). The proportion of cells in the G0/G1 phase significantly increased, whereas the proportion of cells in the $S$ phase significantly decreased (Figure 3B).

\section{Effect of lncRNA FTX on the expression of EMT and PI3K/Akt signaling pathway-related proteins in EESC}

To further investigate the effect of the molecular mechanism of lncRNA FTX on the cellular function of EESC, we first detected the expression of EMT-related proteins in EESC of each group by Western blot. The results showed that the protein expression of E-cadherin significantly decreased, and the protein expression levels of vimentin, $\mathrm{N}$-cadherin, and ZEB1 significantly increased in the cells of the si-FTX group compared with the si-NC group $(\mathrm{P}<0.05)$ (Figure $4 A$ ). Compared with the pc-NC group, the protein expression of E-cadherin significantly increased, and the protein expression levels of vimentin, $\mathrm{N}$-cadherin, and ZEB1 significantly decreased in the cells of the pc-FTX group $(\mathrm{P}<0.05)$. It has been noted that PI3K/Akt signaling pathway activation is important for the development of EMs (14). Therefore, we detected the PI3K/Akt signaling pathwayrelated protein expression. The results showed that the expression of p-PI3K/PI3K and p-AKT/AKT significantly increased in the cells of the si-FTX group compared with the si-NC group $(\mathrm{P}<0.05)$ (Figure $4 B)$. The expressions of $\mathrm{p}-\mathrm{PI} 3 \mathrm{~K} / \mathrm{PI} 3 \mathrm{~K}$ and $\mathrm{p}$-AKT/AKT significantly decreased in the cells of the pc-FTX group compared with the pc-NC group $(\mathrm{P}<0.05)$. These results confirmed that lncRNA FTX is involved in regulating EMT transformation in EESC and in PI3K/Akt signaling pathway activation.

\section{Discussion}

Although there are many theories about the etiology 

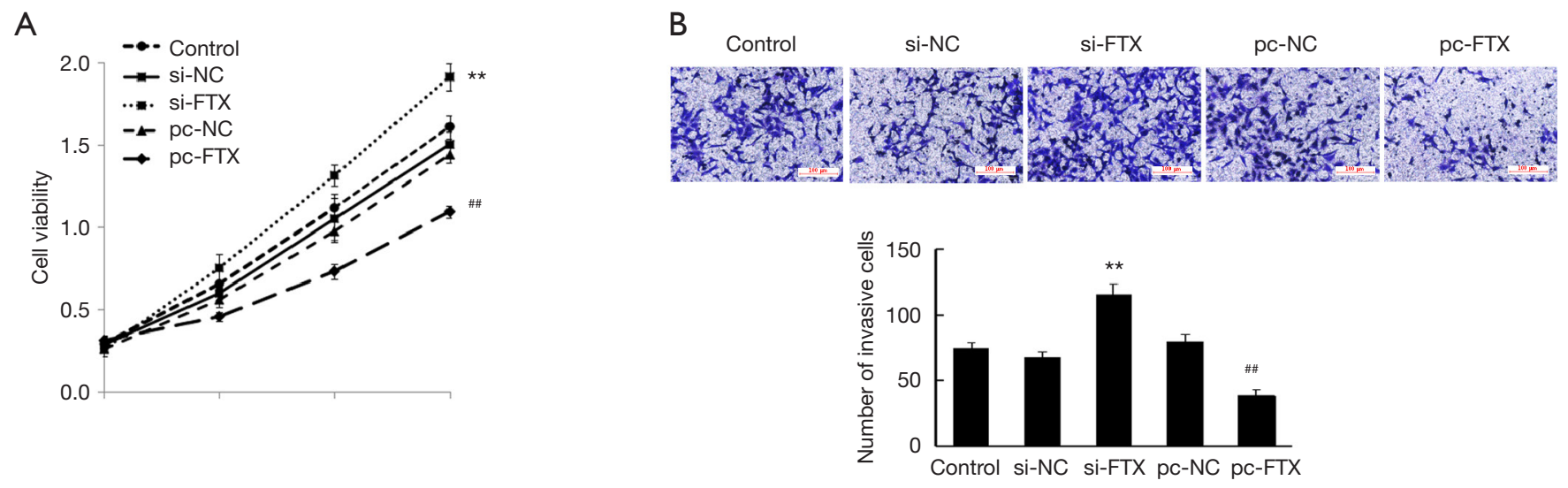

C
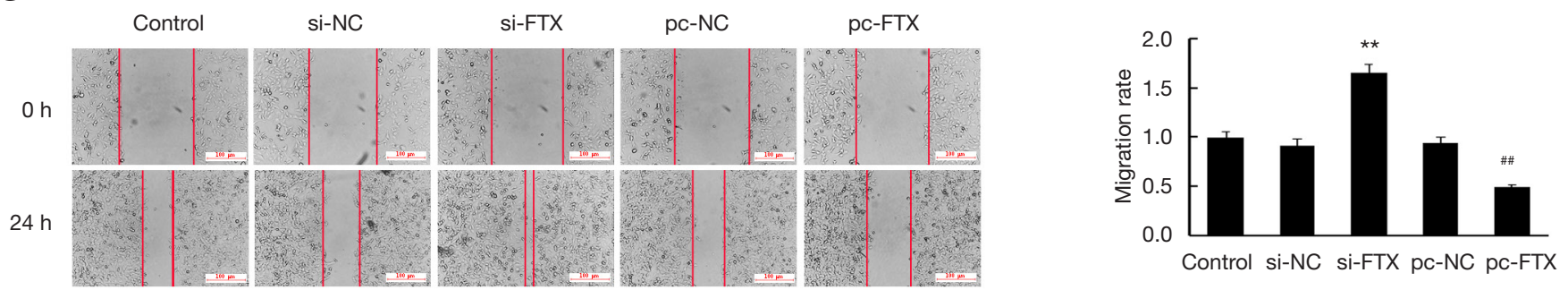

Figure 2 Long non-coding RNA 5 prime to Xist (lncRNA FTX) promotes the proliferation, invasion, and migration of ectopic endometrial stromal cells. (A) Cell Counting Kit-8 was used to detect the proliferation of cells in each group after transfecting for 24, 48, and 72 h, respectively. (B) Transwell assay was used to detect the invasion of cells in each group after transfection (scale $=100 \mu \mathrm{m})$. (C) Scratch assay was used to detect the migration of cells in each group after transfection (scale $=100 \mu \mathrm{m})$. ${ }^{*}$, compared with negative siRNA (si-NC) group, and $\mathrm{P}<0.01$; ${ }^{\#}$, compared with pcDNA3.1 empty vector (pc-NC) group, and $\mathrm{P}<0.01$. si-NC, EESC transfected with negative siRNA; siFTX, lncRNA FTX siRNA; pc-NC, pcDNA3.1 empty vector; pc-FTX, overexpressed pcDNA3.1-lncRNA FTX.

of EMs, there is still no consensus on the pathogenesis of EMs. With continuous improvement in highthroughput sequencing and gene chip technology in recent years, studies are increasingly linking lncRNAs to the regulation of a cellular physiological metabolism. It has been noted that lncRNA FTX could inhibit the proliferation and metastasis of hepatocellular carcinoma by binding Minichromosome maintenance protein 2 and miR-374a (15), regulating cardiomyocyte apoptosis by targeting miR-29b-1-5p and Bcl212 (16), and inhibiting the proliferation and migration of osteosarcoma by regulating miR-320a/Thioredoxin Reductase 1 (17). IncRNA FTX is a key lncRNA in regulating the proliferation, migration, and apoptosis of cancer cells; however, the question remains as to whether IncRNA FTX has a corresponding effect on EMs, which is known as "undead cancer". In the present study, using clinical sample tissues and in vitro cells, we found that lncRNA FTX was underexpressed in endometrial tissues and in EESC of patients in the EMs group. The results confirmed that IncRNA FTX was involved in the development of EMs. However, the possible function and mechanism of lncRNA FTX in EMs remain unclear.

The main difference between EESC and normal ESC is that the migration and invasion, as well as the growth and EMT of EESC, are enhanced (18). Therefore, we interfered with the expression of IncRNA FTX to study its effect on the molecular functions of EESC. After interference and overexpression of IncRNA FTX, we detected changes in the proliferation, invasion, and migration of EESC. After interfering with the expression of IncRNA FTX, the proliferation, invasion, and migration of EESC significantly increased, and apoptosis decreased. However, the proliferation, invasion, and migration of EESC significantly decreased after the overexpression of lncRNA FTX. The 

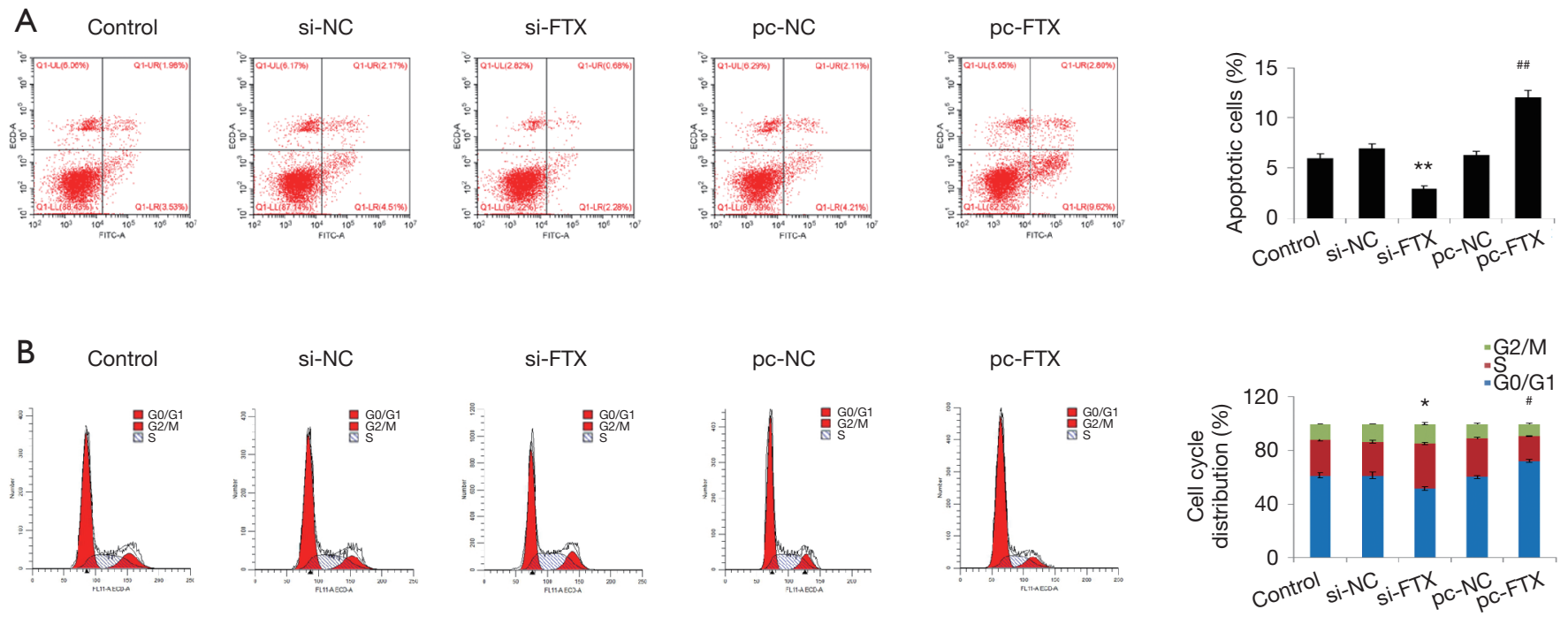

Figure 3 Effect of Long non-coding RNA 5 prime to Xist (lncRNA FTX) on apoptosis and cell cycle arrest of ectopic endometrial stromal cells. (A) Flow cytometry was used to detect the apoptotic level of cells in each group after transfection; (B) flow cytometry was used to detect cell cycle arrest of each group after transfection. ${ }^{*}, \mathrm{P}<0.05$ and ${ }^{* *}, \mathrm{P}<0.01$ compared with negative siRNA (si-NC) group; ${ }^{*}, \mathrm{P}<0.05$ and $^{\#,}, \mathrm{P}<0.01$ compared with pcDNA3.1 empty vector (pc-NC) group. si-NC, EESC transfected with negative siRNA; si-FTX, lncRNA FTX siRNA; pc-NC, pcDNA3.1 empty vector; pc-FTX, overexpressed pcDNA3.1-lncRNA FTX.

flow cytometry results showed that if the expression of lncRNA FTX increased, the apoptotic rate also increased, and lncRNA FTX would lead to the cell cycle arrest of EESC in the G0/G1 phase. However, recent studies have shown that the knockdown of lncRNA FTX inhibits the proliferation, migration, and invasion of renal cancer cells, and results in the cell cycle arrest of renal cancer cells in the G0/G1 phase (19). It has also been reported that lncRNA FTX promotes the progression of gastric cancer by targeting miR-215 (20). The findings of this experiment, combined with those of previous studies, indicate that the role of IncRNA FTX in the process of disease development is 2 sided.

It has been noted that PI3K/Akt signaling pathway activation is important for the development of EMs; it was not only involved in regulating the proliferation, invasion, and migration of EESC, but also affected the EMT of cells (14). However, inhibiting PI3K/Akt signaling pathway activity could have an inhibitory effect on EMs $(21,22)$. EMT has been reported to be a key indicator of the invasive and metastatic process of ectopic endometria and the development of EMs (23). To investigate the effect of lncRNA FTX on EMT, as well as the mechanisms for EESC regulation, EMT-related proteins and PI3K/ Akt signaling pathway-related proteins were detected by Western blot. We found that the overexpression of lncRNA FTX significantly increased the protein expression of E-cadherin, and decreased the protein expression of vimentin, $\mathrm{N}$-cadherin, and ZEB1. The knockdown of lncRNA FTX may result in increased ratios of p-PI3K/ $\mathrm{PI} 3 \mathrm{~K}$ and $\mathrm{p}-\mathrm{Akt} / \mathrm{Akt}$, whereas the overexpression of lncRNA FTX decreased the ratios of $\mathrm{p}-\mathrm{PI} 3 \mathrm{~K} / \mathrm{PI} 3 \mathrm{~K}$ and $\mathrm{p}$-Akt/Akt. These results suggest that the overexpression of lncRNA FTX can inhibit the activity of PI3K/Akt signaling pathway as well as the proliferation, invasion and EMT activity of EESCs in the clinical treatment, and prevent further deterioration of endometriosis.

\section{Conclusions}

The decreased expression of lncRNA FTX is associated with the development of EMs. The overexpression of lncRNA FTX can reduce EMs-induced proliferation, invasion, metastasis, and EMPT of ESC by inhibiting PI3K/Akt signaling pathway activity. Therefore, increasing the expression of IncRNA FTX could be used as a new treatment modality for EMs. However, because there are many cellular activities that lncRNA FTX can participate in, and because its interaction with target genes and target proteins is very complicated, the exact mechanism of 

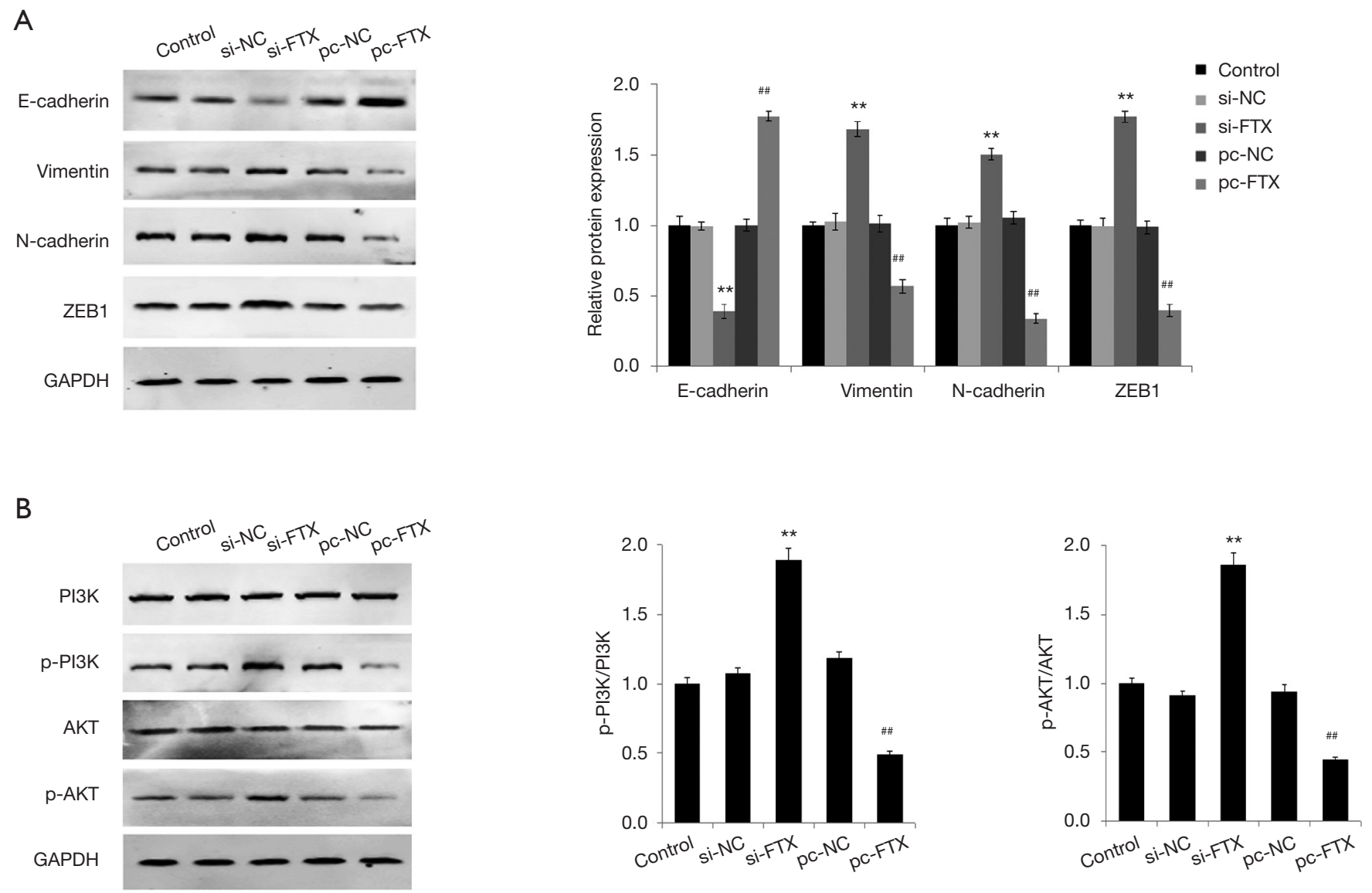

Figure 4 Effect of Effect of Long non-coding RNA 5 prime to Xist (lncRNA FTX) on the expression of epithelial-mesenchymal transition and PI3K/Akt signaling pathway-related protein in ectopic endometrial stromal cells (EESC). (A) Western blot was used to detect the expression of E-cadherin, vimentin, N-cadherin, and zinc finger E-box binding homeobox 1 (ZEB1) in the EESC of each group after transfection; (B) Western blot was used to detect the expression of PI3K, p-PI3K, AKT, and p-AKT in the EESC of each group after transfection. **, compared with negative siRNA (si-NC) group, and $\mathrm{P}<0.01$; ${ }^{\# \#}$, compared with pcDNA3.1 empty vector (pc-NC) group, and $\mathrm{P}<0.01$. si-NC, EESC transfected with negative siRNA; si-FTX, lncRNA FTX siRNA; pc-NC, pcDNA3.1 empty vector; pc-FTX, overexpressed pcDNA3.1-lncRNA FTX.

lncRNA FTX in regulating the expression of EMT-related proteins and inhibiting PI3K/Akt signaling pathway activity needs to be further explored.

\section{Acknowledgments}

Funding: None.

\section{Footnote}

Reporting Checklist: The authors have completed the MDAR reporting checklist. Available at http://dx.doi.org/10.21037/ atm-20-6810
Data Sharing Statement: Available at http://dx.doi, org/10.21037/atm-20-6810

Conflicts of Interest: All authors have completed the ICMJE uniform disclosure form (available at http://dx.doi. org/10.21037/atm-20-6810). The authors have no conflicts of interest to declare.

Ethical Statement: The authors are accountable for all aspects of the work in ensuring that questions related to the accuracy or integrity of any part of the work are appropriately investigated and resolved. All procedures performed in this study involving human participants were 
in accordance with the Declaration of Helsinki (as revised in 2013). All study procedures were approved by the Research Ethics Committee of Beijing Tongren Hospital. Informed consent was taken from all the patients.

Open Access Statement: This is an Open Access article distributed in accordance with the Creative Commons Attribution-NonCommercial-NoDerivs 4.0 International License (CC BY-NC-ND 4.0), which permits the noncommercial replication and distribution of the article with the strict proviso that no changes or edits are made and the original work is properly cited (including links to both the formal publication through the relevant DOI and the license). See: https://creativecommons.org/licenses/by-nc-nd/4.0/.

\section{References}

1. Parasar P, Ozcan P, Terry KL. Endometriosis: Epidemiology, Diagnosis and Clinical Management. Curr Obstet Gynecol Rep 2017;6:34-41.

2. de Ziegler D, Borghese B, Chapron C. Endometriosis and infertility: pathophysiology and management. Lancet 2010;376:730-8.

3. Johnson NP, Hummelshoj L; World Endometriosis Society Montpellier Consortium. Consensus on current management of endometriosis. Hum Reprod 2013;28:1552-68.

4. Ferrero S, Vellone VG, Barra F. Pathophysiology of pain in patients with peritoneal endometriosis. Ann Transl Med 2019;7:S8.

5. Nishida M, Nasu K, Ueda T, et al. Endometriotic cells are resistant to interferon-gamma-induced cell growth inhibition and apoptosis: a possible mechanism involved in the pathogenesis of endometriosis. Mol Hum Reprod 2005;11:29-34.

6. Ahn SH, Singh V, Tayade C. Biomarkers in endometriosis: challenges and opportunities. Fertil Steril 2017;107:523-32.

7. Luo H, Yang H, Lin $\mathrm{Y}$, et al. LncRNA and mRNA profiling during activation of tilapia macrophages by HSP70 and Streptococcus agalactiae antigen. Oncotarget 2017;8:98455-70.

8. Rinn JL, Chang HY. Genome regulation by long noncoding RNAs. Annu Rev Biochem 2012;81:145-66.

9. Backofen R, Vogel T. Biological and bioinformatical approaches to study crosstalk of long-non-coding RNAs and chromatin-modifying proteins. Cell Tissue Res 2014;356:507-26.

10. Wu T, Du Y. LncRNAs: From Basic Research to Medical Application. Int J Biol Sci 2017;13:295-307.

11. Shi S, Yang J, Fan W, et al. Effects of LncRNA MALAT1 on microangiopathy and diabetic kidney disease in diabetic rats by regulating ERK/MAPK signaling pathway. Minerva Med 2020;111:184-6.

12. Huang H, Zhu Z, Song Y. Downregulation of lncrna uca1 as a diagnostic and prognostic biomarker for ovarian endometriosis. Rev Assoc Med Bras (1992) 2019;65:336-41.

13. La Y, He X, Zhang L, et al. Comprehensive Analysis of Differentially Expressed Profiles of mRNA, lncRNA, and circRNA in the Uterus of Seasonal Reproduction Sheep. Genes (Basel) 2020;11:301.

14. Madanes D, Bilotas MA, Bastón JI, et al. PI3K/AKT pathway is altered in the endometriosis patient's endometrium and presents differences according to severity stage. Gynecol Endocrinol 2020;36:436-40.

15. Liu F, Yuan JH, Huang JF, et al. Long noncoding RNA FTX inhibits hepatocellular carcinoma proliferation and metastasis by binding MCM2 and miR-374a. Oncogene 2016;35:5422-34.

16. Long B, Li N, Xu XX, et al. Long noncoding RNA FTX regulates cardiomyocyte apoptosis by targeting miR29b-1-5p and Bc1212. Biochem Biophys Res Commun 2018;495:312-8.

17. Huang $\mathrm{S}, \mathrm{Zhu} \mathrm{X}, \mathrm{Ke} \mathrm{Y}$, et al. LncRNA FTX inhibition restrains osteosarcoma proliferation and migration via modulating miR-320a/TXNRD1. Cancer Biol Ther 2020;21:379-87.

18. Liu Y, Lu C, Fan L, et al. MiR-199a-5p targets ZEB1 to inhibit the epithelial-mesenchymal transition of ovarian ectopic endometrial stromal cells via PI3K/Akt/ mTOR signal pathway in vitro and in vivo. Reprod Sci 2020;27:110-8.

19. He X, Sun F, Guo F, et al. Knockdown of long noncoding RNA FTX inhibits proliferation, migration, and invasion in renal cell carcinoma cells. Oncol Res 2017;25:157-66.

20. Yang $Y$, Zhang J, Chen X, et al. LncRNA FTX sponges miR-215 and inhibits phosphorylation of vimentin for promoting colorectal cancer progression. Gene Ther 2018;25:321-30.

21. Barra F, Ferro Desideri L, Ferrero S. Inhibition of PI3K/ AKT/mTOR pathway for the treatment of endometriosis. 
Page 10 of 10

Br J Pharmacol 2018;175:3626-7.

22. Jing $W$, Yan $W$, Du X, et al. Sulforaphane Attenuates Endometriosis in Rat Models Through Inhibiting PI3K/Akt Signaling Pathway. Journal of Clinical and Experimental Medicine 2019;17:155-8.

Cite this article as: Wang $\mathrm{H}, \mathrm{Ni} \mathrm{C}$, Xiao W, Wang S. Role of lncRNA FTX in invasion, metastasis, and epithelialmesenchymal transition of endometrial stromal cells caused by endometriosis by regulating the PI3K/Akt signaling pathway. Ann Transl Med 2020;8(22):1504. doi: 10.21037/atm-20-6810

\section{Wang et al. The effect and mechanism of IncRNA FTX in EMs}

23. Zheng J, Luo X, Bao J, et al. Decreased Expression of HOXA10 May Activate the Autophagic Process in Ovarian Endometriosis. Reprod Sci 2018;25:1446-54.

(English Language Editor: R. Scott) 